\title{
Elevated expression of chemokine C-C ligand 2 in stroma is associated with recurrent basal- like breast cancers
}

\author{
Min $\mathrm{Yao}^{1}$, Elaine $\mathrm{Yu}^{1}$, Vincent Staggs ${ }^{2}$, Fang Fan $^{1}$ and Nikki Cheng ${ }^{1}$ \\ ${ }^{1}$ Department of Pathology and Laboratory Medicine, University of Kansas Medical Center, Kansas City, KS, \\ USA and ${ }^{2}$ Health Services and Outcomes Research, Children's Mercy Hospitals and Clinics, Kansas City, \\ $M O, U S A$
}

\begin{abstract}
Despite advances in treatment, up to $30 \%$ of breast cancer patients experience disease recurrence accompanied by more aggressive disease and poorer prognosis. Treatment of breast cancer is complicated by the presence of multiple breast cancer subtypes, including: luminal, Her2 overexpressing, and aggressive basal-like breast cancers. Identifying new biomarkers specific to breast cancer subtypes could enhance the prediction of patient prognosis and contribute to improved treatment strategies. The microenvironment influences breast cancer progression through expression of growth factors, angiogenic factors and other soluble proteins. In particular, chemokine $\mathrm{C}-\mathrm{C}$ ligand 2 (CCL2) regulates macrophage recruitment to primary tumors and signals to cancer cells to promote breast tumor progression. Here we employed a software-based approach to evaluate the prognostic significance of CCL2 protein expression in breast cancer subtypes in relation to its expression in the epithelium or stroma or in relation to fibroblast-specific protein 1 (Fsp1), a mesenchymal marker. Immunohistochemistry analysis of tissue microarrays revealed that CCL2 significantly correlated with Fsp1 expression in the stroma and tumor epithelium of invasive ductal carcinoma. In the overall cohort of invasive ductal carcinomas $(n=427)$, CCL2 and Fsp1 expression in whole tissues, stroma and epithelium were inversely associated with cancer stage and tumor size. When factoring in molecular subtype, stromal CCL2 was observed to be most highly expressed in basal-like breast cancers. By Cox regression modeling, stromal CCL2, but not epithelial CCL2, expression was significantly associated with decreased recurrence-free survival. Furthermore, stromal CCL2 (HR $=7.51$ $P=0.007)$ was associated with a greater hazard than cancer stage $(H R=2.45, P=0.048)$ in multivariate analysis. These studies indicate that stromal CCL2 is associated with decreased recurrence-free survival in patients with basal-like breast cancer, with important implications on the use of stromal markers for predicting patient prognosis. Modern Pathology (2016) 29, 810-823; doi:10.1038/modpathol.2016.78; published online 29 April 2016
\end{abstract}

Breast cancer is the most common form of cancer diagnosed in women, with over 1.3 million new cases diagnosed annually in the world. ${ }^{1}$ Invasive ductal carcinomas comprise $80 \%$ of all invasive breast cancer cases and are commonly treated with a combination of radiation, chemotherapy, hormone therapy and targeted Her2 therapy. ${ }^{2-4}$ Despite advances in treatment, disease recurrence remains high, up to $56 \%$ for patients diagnosed with stage III

Correspondence: Dr N Cheng, PhD, Department of Pathology and Laboratory Medicine, University of Kansas Medical Center, 3901 Rainbow Boulevard, WHE 1020, MS 3003, Kansas City, KS 66160, USA.

E-mail: ncheng@kumc.edu

Received 1 December 2015; revised 10 March 2016; accepted 12 March 2016; published online 29 April 2016 breast cancer. Metastatic occurrence often accompanies disease relapse, contributing to an $80 \%$ mortality rate, and estimated 450000 deaths worldwide. ${ }^{1,5,6}$ Treatment of breast cancer is complicated by the presence of different molecular subtypes. These subtypes include: luminal A, luminal B, Her2 overexpressing (Her2+), and basal-like breast cancers. Histologically, these subtypes are classified by the status of estrogen receptor (ER), progesterone receptor (PR), Her2 and cell proliferative index. ${ }^{7-10}$ Conventional prognostic indicators such as grade and stage do not accurately predict patient prognosis for all of the different molecular subtypes, ${ }^{11,12}$ indicating the need to identify prognostic factors unique to each subtype.

The stroma greatly influences breast cancer progression and therapeutic responsiveness. Desmoplasia is a 
form of reactive stroma and is characterized by the accumulation of fibroblasts. Desmoplasia is significantly associated with invasive breast cancer and decreased relapse-free and overall survival. ${ }^{13-15}$ Although uniform in their spindle-shaped appearance, fibroblasts may be identified by the expression of several different mesenchymal markers, including distinct non-overlapping markers such as $\alpha$-smooth muscle actin and fibroblast-specific protein 1 (Fsp1). ${ }^{16-18}$ As a key cellular component of the microenvironment, fibroblasts influence the growth and progression of breast cancers directly and indirectly, through secretion of growth factors, matrix proteins and angiogenic factors, as demonstrated through animal studies. ${ }^{19-21}$ In tumor-bearing mice, doxorubicin treatment of prostate and colon cancers enhance fibroblast secretion of cytokines associated with development of drug resistance. ${ }^{22,23}$ In co-culture studies, carcinoma-associated fibroblasts promote invasion of basal-like breast cancer cells and growth of Luminal breast cancer cells, ${ }^{24}$ indicating different biological functions for carcinoma-associated fibroblasts of different breast cancer subtypes. Gene profiling studies of breast cancer stroma have identified gene signatures that associate with poor clinical outcome. ${ }^{25,26}$ However, the use of specific stromal markers as reliable predictors of patient prognosis remains to be validated.

Recent studies demonstrate that the stroma of invasive breast cancers express high levels of chemokine C-C ligand 2 (CCL2). CCL2 belongs to a family of chemotactic cytokines $(8 \mathrm{kDa})$, which bind to $G$ protein-coupled receptors to regulate recruitment of macrophages during normal physiologic responses, such as wound healing. ${ }^{27-29}$ CCL2 modulates macrophage recruitment to breast tumors and also signals to breast cancer cells to modulate tumor survival and invasion..$^{30-33}$ Antibody neutralization of CCL2 inhibits growth and invasion of breast tumor xenografts. ${ }^{32-34}$ Previous studies have primarily examined CCL2 expression for its association with macrophages in breast cancer and patient prognosis and have reported different results. ${ }^{32,35,36}$ In previous studies, we demonstrated an important biological role for CCL2 overexpression in breast cancer cells and in fibroblasts in regulating tumor progression. ${ }^{34,37}$ These studies suggest that multiple factors could affect the prognostic relevance of CCL2 expression in breast cancer.

In these studies, we employed several novel approaches to evaluate the prognostic significance of CCL2 expression in breast cancer. Here we employed a software-based approach to evaluate the prognostic significance of CCL2 protein expression in breast cancer subtypes in relation to its expression in the epithelium or stroma or in relation to Fsp1, a mesenchymal marker. Immunohistochemistry analysis of tissue microarrays revealed that CCL2 significantly correlated with Fsp1 expression in the stroma and tumor epithelium of invasive ductal carcinoma. In the overall breast cancer cohort,
CCL2 and Fsp1 expression in whole tissues, stroma and epithelium were inversely associated with cancer stage and tumor size. When factoring in molecular subtype, stromal CCL2 was observed to be most highly expressed in basal-like breast cancers. By Cox regression modeling, stromal CCL2, but not stromal Fsp1 expression was significantly associated with decreased recurrence-free survival. Furthermore, stromal CCL2 (HR $=7.51, P=0.007)$ was associated with a greater hazard than cancer stage ( $\mathrm{HR}=2.45, P=0.048)$. Our studies have important implications on the use of stromal markers for predicting prognosis of patients with basal-like breast cancer.

\section{Materials and methods}

\section{Patient Samples}

A total of 10 prognostic tissue microarray slides (5 slides in duplicate) were obtained from the National Cancer Institute Cancer Diagnostics Program. Each tissue microarray slide contained 90-100 breast cancer core samples and 5 normal breast tissue cores, 3 fibroadenoma cores and 20 control core samples comprised of prostate, colon and salivary gland and endometrium tissues. Deidentified carcinoma samples were collected from patients who were diagnosed with breast cancer between 1985 and 1997, prior to adjuvant therapy. The majority of those patients $(85 \%)$ received adjuvant radiation, chemotherapy or hormone therapy or a combination of therapies. The mean followup time was 104 months, with a maximal follow-up time of 276 months. Pathology reports included information on: clinical diagnosis, treatment regimen, age, stage, tumor size, grade, lymph node status, mitotic score, and intensity of biomarker staining for ER, PR and Her2. Patient outcome included information on: recurrence (metastatic or non-metastatic), survival, and follow-up time.

An additional two tissue microarray slides containing 5 de-identified normal breast tissue samples and 32 core samples of stages I-IV breast ductal carcinoma were obtained in duplicate from the Biospecimen Core Repository at the University of Kansas Medical Center. The University of Kansas Medical Center tissue microarray included pathology information on: age, stage, grade, tumor size, Ki67 expression, and intensity of biomarker staining for ER, PR and Her2. Outcome information included: status on survival, recurrence, and number of recurrences. Treatment information was not available.

A total of 427 breast tumor samples were analyzed. The clinical pathological features of both data sets are summarized (Table 1). When the patient data sets were combined, the mean age of patients was 59 years, with a mean follow-up time of 8.7 years. 
Table 1 Clinico-pathological features of tumor samples from the National Cancer Institute and University of Kansas Medical Center cohorts combined

\begin{tabular}{|c|c|}
\hline Factors & Sample size (\% of total) \\
\hline \multicolumn{2}{|l|}{ Age (years) } \\
\hline$<50$ & $130(30 \%)$ \\
\hline$\geq 50$ & $297(70 \%)$ \\
\hline \multicolumn{2}{|l|}{ Histology type } \\
\hline Ductal & $388(91 \%)$ \\
\hline Lobular & $38(9 \%)$ \\
\hline Unknown & $1(0.2 \%)$ \\
\hline \multicolumn{2}{|l|}{ T stage } \\
\hline $\mathrm{T} 1$ & $90(21 \%)$ \\
\hline $\mathrm{T} 2$ & $196(46 \%)$ \\
\hline T3 & $88(21 \%)$ \\
\hline $\mathrm{T} 4$ & $42(10 \%)$ \\
\hline Unknown & $11(3 \%)$ \\
\hline \multicolumn{2}{|l|}{$N$ stage } \\
\hline No & $92(22 \%)$ \\
\hline N1 & $266(63 \%)$ \\
\hline N2 & $54(13 \%)$ \\
\hline N3 & $3(1 \%)$ \\
\hline Unknown & $12(3 \%)$ \\
\hline \multicolumn{2}{|l|}{ Grade } \\
\hline I & $61(14 \%)$ \\
\hline II & $197(46 \%)$ \\
\hline III & 167 (39\%) \\
\hline Unknown & $2(0.5 \%)$ \\
\hline \multicolumn{2}{|l|}{ ER status } \\
\hline Negative & $145(34 \%)$ \\
\hline Positive & $271(63 \%)$ \\
\hline Unknown & $11(3 \%)$ \\
\hline \multicolumn{2}{|l|}{ PR status } \\
\hline Negative & $168(39 \%)$ \\
\hline Positive & 247 (58\%) \\
\hline Unknown & $12(3 \%)$ \\
\hline \multicolumn{2}{|l|}{ Her2 status } \\
\hline Normal & $339(79 \%)$ \\
\hline Overexpression & $76(18 \%)$ \\
\hline Unknown & $12(3 \%)$ \\
\hline
\end{tabular}

\section{Immunohistochemistry}

Tissue microarray slides were de-paraffinized and rehydrated through a series of xylenes and 100, 95, 7 and $50 \%$ ethanols. For CCL2 immunostaining, slides underwent antigen retrieval through lowpressure cooking in $2 \mathrm{M}$ urea for $2 \mathrm{~min}$. Peroxidases were quenched for $30 \mathrm{~min}$ in PBS containing $3 \%$ $\mathrm{H}_{2} \mathrm{O}_{2}$ and $10 \%$ Methanol and blocked in PBS containing $3 \%$ fetal bovine serum for $1 \mathrm{~h}$. Slides were incubated with goat anti-human CCL2 antibody at a 1:100 dilution (cat no. sc-1304, Santa Cruz Biotechnology, Santa Cruz, CA, USA) overnight in blocking buffer. CCL2 was detected by incubating sections with biotinylated horse anti-goat antibody (Vector Laboratories, Burlingame, CA, USA, BH-9500, 1:1000) in room temperature for $2 \mathrm{~h}$ and then with strepatividin-peroxidase (cat no.
PK-6100, Vector Laboratories). For Fsp1 immunostaining, slides underwent antigen retrieval through low-pressure cooking in $10 \mathrm{mM}$ sodium citrate $\mathrm{pH}$ 6.8 for 2 min. After peroxidase quenching and blocking, slides were incubated with rabbit antiFsp1 at a 1:3 dilution (cat no. ab-27427, Abcam, Cambridge, MA, USA) in blocking buffer overnight and then with peroxidase-conjugated goat anti-rabbit antibody (cat no. 611-1302, Rockland Immunochemicals, Pottstown, PA, USA) for $2 \mathrm{~h}$ at room temperature. Expression of CCL2 and Fsp1 proteins were detected with 3,3'-diaminobenzidine (DAB) substrate (Vector Laboratories, SK-4100). Slides were counterstained with Harris's hematoxylin, dehydrated and mounted with Cytoseal (cat no. 8312-4, Thermo Fisher Scientific, Lenexa, KS, USA). The specificity of the CCL2 or Fsp1 antibodies were determined by staining in the presence or absence of $1 \mu \mathrm{g}$ (5foldexcess) of CCL2-blocking peptide (cat no. 1304P, Santa Cruz Biotechnology) or Fsp1 recombinant protein (cat no.ab83650, Abcam, Cambridge, UK).

For immunofluorescence staining, CCL2 expression was detected through incubation with secondary donkey anti-goat conjugated to Alexa Flour-488 at a 1:1000 dilution (cat no. R37118, Thermo Fisher Scientific). To detect Fsp1, slides were incubated with secondary rabbit biotinylated antibodies, conjugated to strepatividin-Alexa Flour-568 (cat no. s-11226, Thermo Fisher Scientific). Slides were counterstained with DAPI and mounted in PBS containing $50 \%$ glycerol.

\section{Image Quantification}

Images of immunohistochemistry staining were acquired at $\times 10$ magnification using a Motic AE31 inverted microscope with Infinity 2-1 x color digital camera. Captured images represented $80-100 \%$ of the whole section. Software analysis of biomarker expression in breast tissues was performed using methods previously described. ${ }^{38}$ Images were first imported into Adobe Photoshop. Color and exposure of images were normalized using auto-contrast. Total DAB staining was selected using the Magic Wand tool in the Color Range Window, with a specificity range of 66. Selected pixels were copied to a new window and saved on a separate file. Images were opened in the Image J software (NIH) and converted to gray scale. Background pixels resulting from luminosity of brightfield images were removed by threshold adjustment. Images were the subject to particle analysis. DAB staining and total areas were expressed as particle area values of arbitrary units. Positive DAB values were normalized to total area values. To quantify stromal or epithelial staining, tumor epithelium was distinguished from the stroma by differences in nuclear and cellular morphology and tissue architecture. In Adobe Photoshop, the lasso tool was to select and crop out stroma or epithelium in the captured image. The stromal 
tissues were labeled as 'total stromal area' while the epithelial tissues were labeled as 'total epithelial area'. DAB staining, identified as brown, was selected using the Magic Wand tool, copied to a new window and saved as a separate file. Images were opened in the Image J software (NIH) and quantified. Positive epithelial DAB values were normalized to total epithelial values. Positive stromal DAB values were normalized to total stromal values.

\section{Manual Scoring of Tissues}

A subset of samples immunostained for CCL2 $(n=46)$ and Fsp1 $(n=15)$ were provided to a clinical pathologist for blinded scoring. As cells in each sample appeared to express CCL2 at varying intensities, the samples were scored weak or strong. The pathologist observed cells positive or negative for Fsp1 expression among samples. Thus Fsp1 was scored by quantifying the number of positively expressing cells and normalizing to the total number of cells.

\section{Statistical Analysis}

Sample populations did not fit a Gaussian distribution and were observed to be uneven. The uneven characteristic was due to two factors. Information on some prognostic factors (ER, PR, Her2, tumor grade) was not provided for all patients from the University of Kansas Medical Center and the National Cancer Institute cohorts. In addition, some tissue samples on the tissue microarrays did not adhere to the slide during staining. As such, protein expression values and their relationships to clinical data were analyzed using non-parametric methods. Statistical analyses were performed using the Graphpad software and SAS.

The University of Kansas Medical Center cohort was combined with the National Cancer Institute cohort. Examination of the overall cohort refers to all samples of invasive ductal carcinoma obtained from the University of Kansas Medical Center and the National Cancer Institute. Comparison of two groups with discrete variables was performed using Wilcoxon two-sample test. Tests for association between CCL2 and Fsp1 were performed using Spearman correlation analysis. Analysis of three or more groups was performed using Kruskal-Wallis test with Dunn's post-hoc comparison between groups. Cox regression model for non-proportional hazards was used for univariate and multivariate analysis. Expression levels of CCL2 and Fsp1 in the Cox regression model were transformed by multiplying values by 10 so that values were $>1$. Because of the small number of non-metastatic recurrences $(n=38$ out of 138 recurrent cases), we analyzed recurrencefree survival based on the overall number of recurrent cases, non-metastatic and metastatic recurrences combined. Statistical significance was determined by $P<0.05$. Factors approaching significance were further selected for multivariate analysis. ${ }^{*} P<0.05, \quad{ }^{*} P<0.01$ and $* * * P<0.001$, unless otherwise specified.

\section{Ethics Statements}

The tissues collected for these studies were de-identified and classified as 'exempted' according to regulations set forth by the Human Research Protection Program (ethics committee) at the University of Kansas Medical Center (\#080193). Written informed consent for tissue collection was obtained by the Biospecimen Core Repository. Tissue samples were de-identified by the National Cancer Institute Diagnostics Program and the Biospecimen Core Repository prior to distribution to the investigators. Existing medical records were used in compliance with the regulations of the University of Kansas Medical Center and National Cancer Institute. These regulations are aligned with the World Medical Association Declaration of Helsinki.

\section{Results}

\section{CCL2 and Fsp1 are Co-Expressed in Breast Cancer}

To characterize the expression patterns of CCL2 and Fsp1 in breast cancer, we performed immunohistochemistry staining on breast tissue microarrays obtained from the National Cancer Diagnostics Program and the University of Kansas Medical Center Biospecimen Core Repository. CCL2 and Fsp1 were expressed in both the epithelial and stromal tissues. CCL2 staining appeared membranous and cytoplasmic. Fsp1 appeared to be expressed in the cytoplasm and nucleus. To control for non-specific staining, sections were incubated with secondary antibodies only or with blocking peptides to compete with anti-CCL2 or anti-Fsp1. As shown in Supplementary Figure S1, incubation with secondary antibody only or with blocking peptides resulted in negative DAB staining, demonstrating specificity of CCL2 and Fsp1 antibodies. To quantify protein expression, we utilized an Image J softwarebased approach. ${ }^{38}$ This method is low cost, freely available and would result in yield continuous values, possibly reducing the subjectivity commonly found in scoring. ${ }^{39}$ Expression patterns were compared among normal breast tissues, fibroadenoma tissues, which are non-malignant breast lesions, and invasive breast carcinoma tissues. Of the invasive breast cancer cases, 91\% were comprised of invasive ductal carcinoma and $9 \%$ were diagnosed as invasive lobular carcinoma. Expression levels of CCL2 and Fsp1 proteins were detected in the stroma and epithelium of normal breast tissues, fibroadenoma, invasive lobular carcinoma and invasive ductal carcinoma (Figure 1a). When quantified in whole tissues, CCL2 and Fsp1 were most highly expressed in invasive ductal carcinoma tissues, 
a
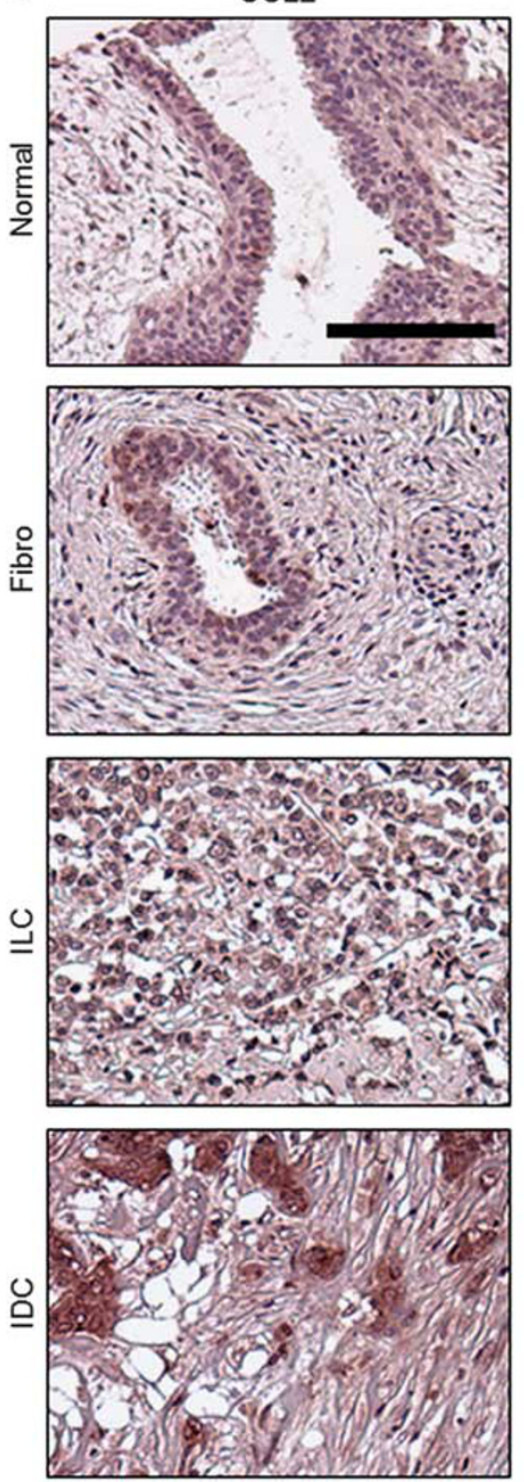
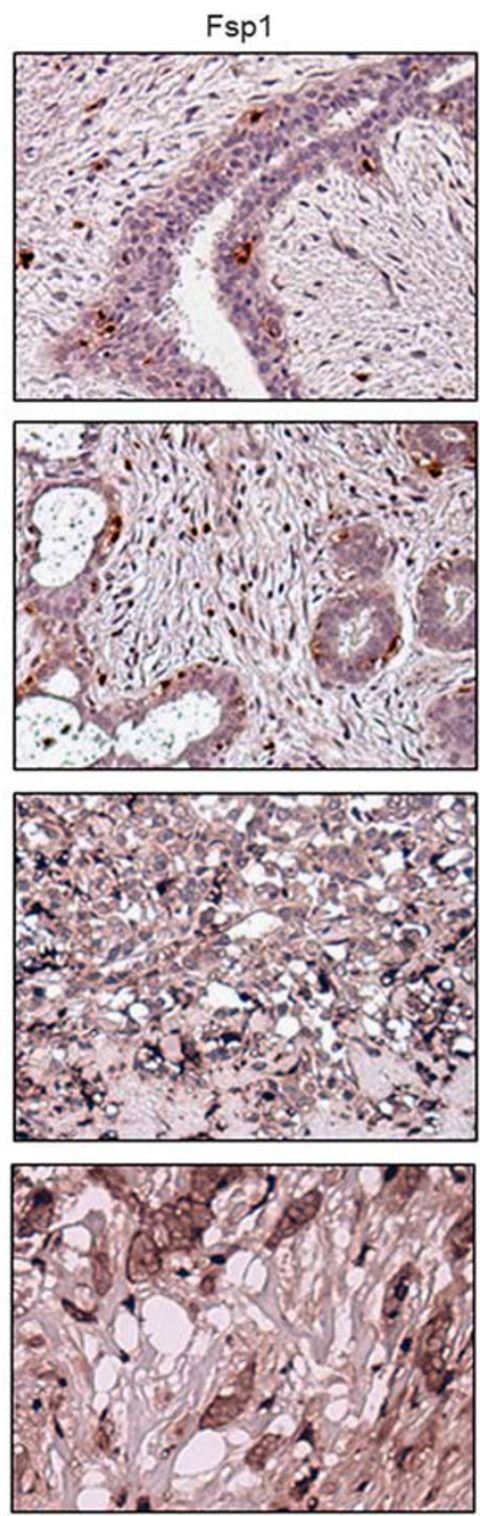

b

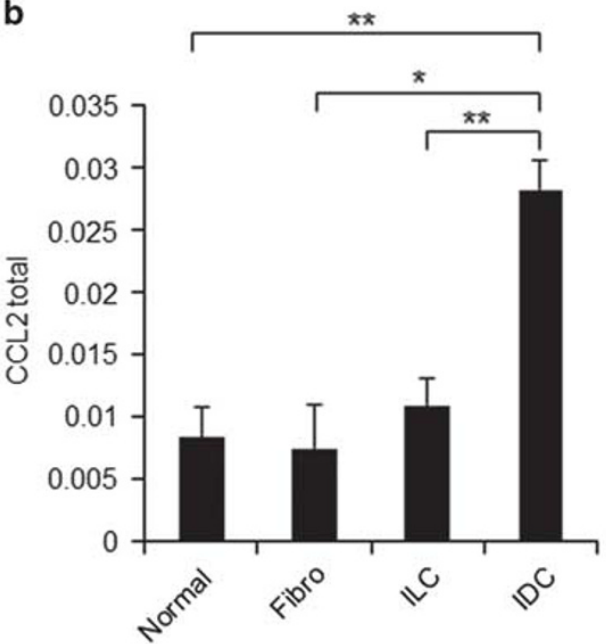

C

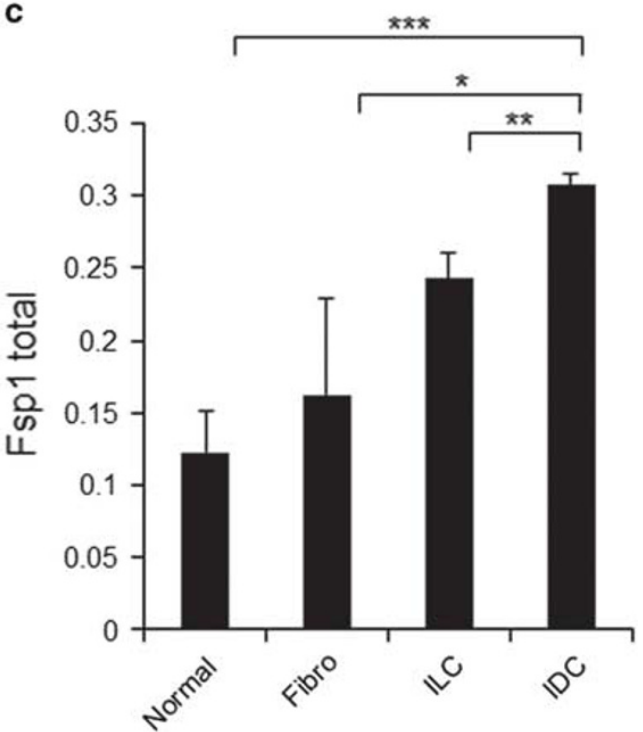

Figure 1 Expression of CCL2 and Fsp1 proteins in breast tissues. Tissue microarrays containing samples of normal breast ( $n=20)$, fibroadenoma (Fibro, $n=9$ ), invasive lobular carcinoma $(n=38)$ and invasive ductal carcinoma (invasive ductal carcinoma, $n=388$ ) breast cancers were immunostained for CCL2 and Fsp1. Expression levels were quantified by Image J; arbitrary units are shown. (a) Representative images of CCL2 and Fsp1 expression. Scale bar=100 microns. Comparison of CCL2 (b) and Fsp1 (c) expression among different groups using Kruskal-Wallis test with Dunn's post-hoc comparison. Statistical significance was determined by ${ }^{*} P<0.05$, $* * P<0.01$ and $* * * P<0.001$

compared with the other tissue types (Figures $1 \mathrm{~b}$ and c). By Spearman correlation analysis, CCL2 was significantly associated with Fsp1 expression in whole tissues, in the stroma and in the epithelium of invasive ductal carcinoma (Figures 2a-c). By coimmunofluorescence staining of invasive ductal carcinoma samples, CCL2 was found to significantly overlap with Fsp1 expression in the stroma and tumor epithelium (Figure 2d). These data demonstrate significant associations between CCL2 and Fsp1 expression in invasive ductal carcinoma.

We compared results between Image J quantification of staining in total or tissue-specific areas with visual (manual) scoring, one of the most common methods for analysis of biomarker expression. ${ }^{39}$ Blinded studies were performed by a clinical pathologist, in which CCL2 and Fsp1 expression levels were visually scored for a subset of samples. These scores were then cross-referenced with Image J values. With respect to epithelial CCL2 expression, weak-to-strong CCL2 was observed among the samples. As seen in Supplementary Figure S2a, samples that were visually scored weak for epithelial CCL2 resulted in low values by Image J analysis. Samples that were visually scored strong resulted in high values by Image J. With respect to Fsp1 expression, the pathologist observed the presence of cells positive or negative for Fsp1 expression in 

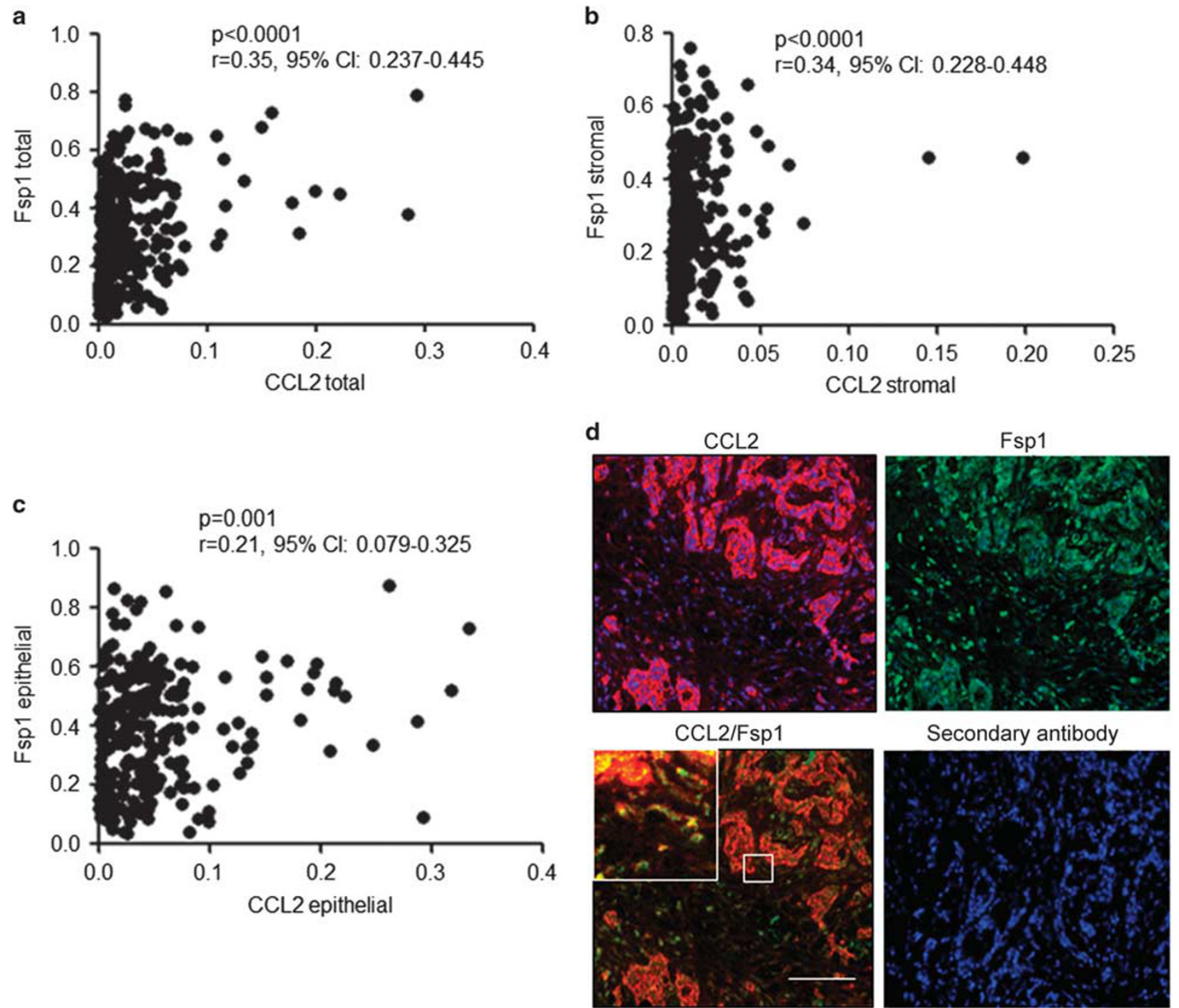

Figure 2 CCL2 is significantly associated with Fsp1 expression in invasive ductal carcinoma Associations between CCL2 and Fsp1 expression in whole tissue (total) (a), stroma (b) or epithelium (c) in invasive ductal carcinoma were analyzed by Spearman correlation analysis. Statistical significance was determined by $P<0.05$. Correlation co-efficient $(r)$ and $95 \%$ confidence interval (95\% CI) are shown. (d) Co-immunofluorescence staining for CCL2 and Fsp1 in invasive ductal carcinoma. Magnified inset shows overlapping staining. Secondary antibody staining is only shown as negative control. Scale bar $=400$ microns.

individual samples. Therefore, the pathologist scored Fsp1 expression by quantifying the ratio of positive cells over the total number of cells. The ratios were then cross-referenced with Image J values. As shown in Supplementary Figure S2b, Image J values significantly correlated to ratios obtained by visual scoring. These data indicate that Image J analysis yields similar results to visual scoring.

\section{CCL2 and Fsp1 Expression do not Significantly} Associate with Patient Outcome in the Overall Cohort

We then analyzed for associations between CCL2 or Fsp1 with commonly used prognostic factors in invasive ductal carcinoma. Whole tissue (total) expression of CCL2 and Fsp1 inversely correlated with overall stage and $\mathrm{T}$ stage (Figures $3 \mathrm{a}$ and $\mathrm{b}$ ). Total Fsp1 but not total CCL2 expression inversely correlated with N stage (Figure 3c). Total expression of CCL2 and Fsp1 was not associated with tumor grade, ER, PR or Her2 status (Supplementary Table S1). Similarly, stromal and epithelial expression of CCL2 and Fsp1 negatively correlated with overall stage and T stage (Supplementary Tables S2 and S3). In contrast to total CCL2 expression, stromal CCL2 expression negatively correlated with Her2 overexpression. In contrast to total Fsp1 expression, epithelial Fsp1 positively correlated with ER and PR status (Supplementary Tables S2 and S3). In summary, these data indicate that CCL2 and Fsp1 inversely associate with stage and tumor size and 

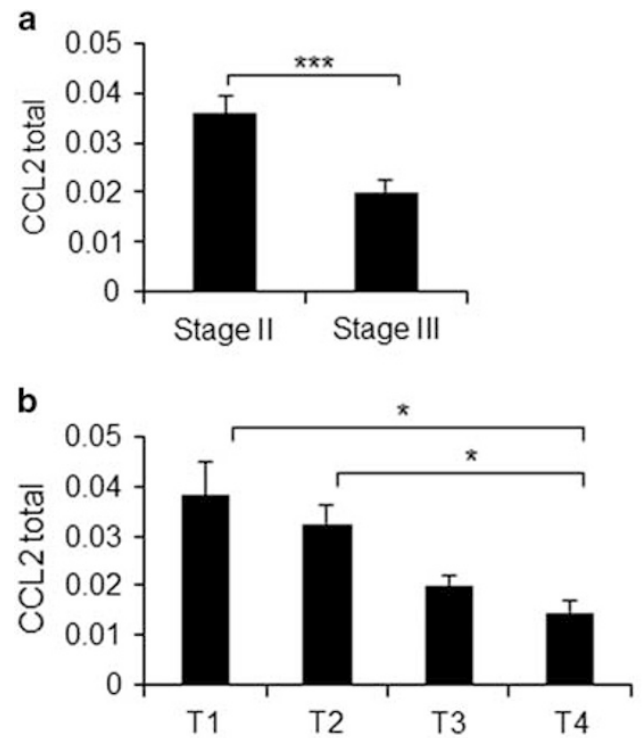

C

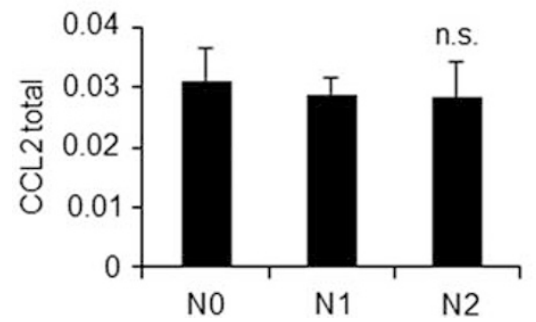

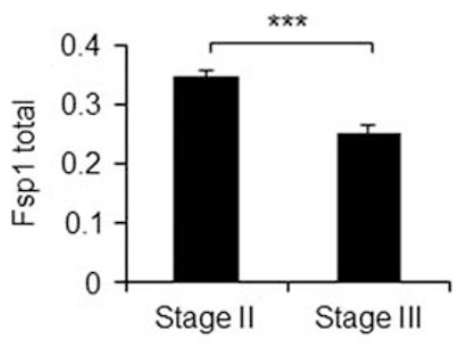
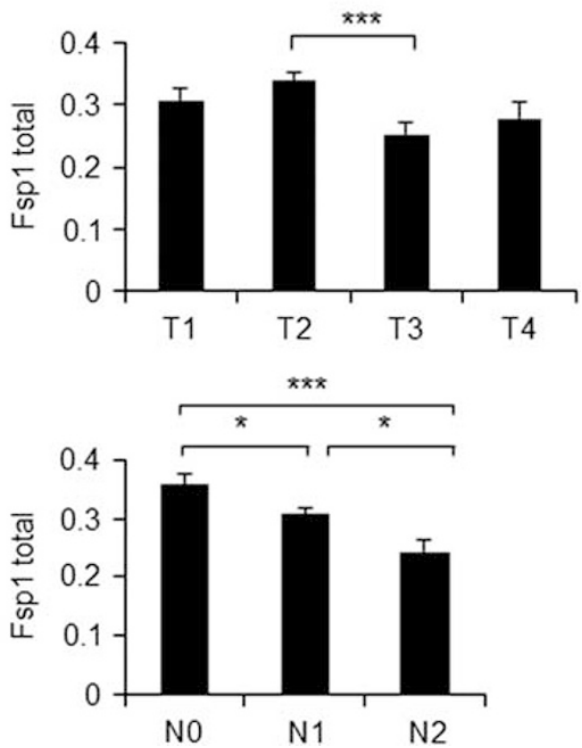

Figure 3 CCL2 and Fsp1 proteins are associated with overall stage and T stage in invasive ductal carcinoma. Total CCL2 and Fsp1 expression were analyzed for associations with overall stage (a), T stage (b) and N stage (c). Statistical analysis was performed using MannWhitney test (overall stage) or Kruskal-Wallis test with Dunn's post-hoc comparison (T stage and N stage). Statistical significance was determined by ${ }^{*} P<0.05 ;{ }^{* *} P<0.001$ and $N S=$ not significant.

that associations with ER, PR and Her2 status are dependent on tissue-specific patterns of expression.

We next determined whether CCL2 and Fsp1 expression were associated with patient outcome. To adjust for multiple variables that might affect the association of CCL2 and Fsp1 expression with patient outcome, univariate Cox regression analysis was first performed for overall survival (Table 2). $\mathrm{T}$ stage, lymph node status and histological grade were associated with decreased overall survival, with hazard ratios of $\geq 1.58$ and $P$-values of $<0.01$. Among adjuvant therapies as possible factors, chemotherapy was associated with decreased overall survival ( $\mathrm{HR}=1.42, P=0.046)$. Radiation therapy and hormone treatment were not significantly associated with overall survival. Among expression of biomarkers, the absence of ER and PR significantly associated with decreased overall survival, with hazard ratios of $\geq 1.84$ and $P$-values $<0.001$. Expression of CCL2 and Fsp1 proteins in whole tissue, stroma and epithelium were not associated with overall survival. We next examined for associations with recurrence-free survival (Table 2). T stage, lymph node status and histological grade were associated with decreased recurrence-free survival, with hazard ratios of $\geq 1.38$ and $P$-values $<0.01$. Chemotherapy, but not antihormonal or radiation therapy, was associated with decreased relapse-free survival with a hazard ratio of 1.43 and $P=0.037$. ER and PR, but not Her2, were associated with decreased recurrence-free survival, with hazard ratios of $\geq 1.70$ and $P$-values of $<0.01$. Expression of CCL2 and Fsp1 proteins in whole tissue, stroma and epithelium were not associated with recurrencefree survival. In summary, CCL2 and Fsp1 expression in invasive ductal carcinoma does not associate with patient outcome.

\section{Stromal CCL2 Associates with Decreased Relapse-Free Survival in Patients with Basal-Like Breast Cancer}

Given the heterogeneity of breast cancer, and some associations with hormone receptor status, it was possible that expression of CCL2 and Fsp1 proteins were associated with breast subtype. Therefore, we compared the expression patterns of CCL2 and Fsp1 in normal breast, luminal A, luminal B, Her2+ and basal-like breast cancers. Luminal A breast cancers were identified as ER+ and/or PR+, Her2 - and low 
Table 2 Univariate Cox regression model on the association of CCL2 and Fsp1 expression with outcome of patients with invasive ductal carcinoma

\begin{tabular}{|c|c|c|c|c|c|c|}
\hline \multirow{2}{*}{ Factors } & \multicolumn{3}{|c|}{ Overall survival } & \multicolumn{3}{|c|}{ Recurrence-free survival } \\
\hline & $H R$ & $95 \% C I$ & $\mathrm{P}$-value & $H R$ & $95 \% C I$ & $\mathrm{P}$-value \\
\hline Age at diagnosis & 1.00 & $0.99,1.02$ & 0.644 & 1.00 & $0.99,1.01$ & 0.965 \\
\hline \multicolumn{7}{|l|}{ T stage } \\
\hline $\mathrm{T} 1$ & 1.00 & & $<0.001$ & 1.00 & & 0.008 \\
\hline $\mathrm{T} 2$ & 1.59 & $0.95,2.67$ & & 1.62 & $1.01,2.58$ & \\
\hline T3 & 3.64 & $2.12,6.23$ & & 2.39 & $1.40,4.07$ & \\
\hline $\mathrm{T} 4$ & 1.97 & $0.97,3.98$ & & 2.30 & $1.20,4.41$ & \\
\hline \multicolumn{7}{|l|}{$N$ stage } \\
\hline No & 1.00 & & 0.002 & 1.00 & & $<0.001$ \\
\hline N1 & 1.58 & $0.98,2.54$ & & 1.38 & $0.89,2.14$ & \\
\hline N2 & 2.89 & $1.61,5.21$ & & 2.99 & $1.72,5.19$ & \\
\hline \multicolumn{7}{|l|}{ Grade } \\
\hline I & 1.00 & & 0.002 & 1.00 & & 0.009 \\
\hline II & 3.79 & $1.64,8.75$ & & 2.24 & $1.18,4.25$ & \\
\hline III & 4.62 & $2.00,10.65$ & & 2.69 & $1.42,5.09$ & \\
\hline \multicolumn{7}{|l|}{ Chemotherapy } \\
\hline No & 1.00 & & 0.046 & 1.00 & & 0.037 \\
\hline Yes & 1.42 & $1.01,2.02$ & & 1.43 & $1.02,2.00$ & \\
\hline \multicolumn{7}{|l|}{ Radiation therapy } \\
\hline No & 1.00 & & 0.513 & 1.00 & & 0.114 \\
\hline Yes & 1.13 & $0.79,1.61$ & & 1.32 & $0.94,1.85$ & \\
\hline \multicolumn{7}{|l|}{ Hormone therapy } \\
\hline No & 1.00 & & 0.635 & 1.00 & & 0.563 \\
\hline Yes & 0.92 & $0.65,1.30$ & & 1.10 & $0.79,1.53$ & \\
\hline \multicolumn{7}{|l|}{ ER status } \\
\hline Positive & 1.00 & & $<0.001$ & 1.00 & & 0.001 \\
\hline Negative & 1.92 & $1.37,2.70$ & & 1.75 & $1.26,2.44$ & \\
\hline \multicolumn{7}{|l|}{ PR status } \\
\hline Positive & 1.00 & & $<0.001$ & 1.00 & & 0.002 \\
\hline Negative & 1.84 & $1.32,2.58$ & & 1.70 & $1.22,2.37$ & \\
\hline \multicolumn{7}{|l|}{ Her2 status } \\
\hline Normal & 1.00 & & 0.127 & 1.00 & & 0.134 \\
\hline Overexpression & 1.37 & $0.91,2.06$ & & 1.35 & $0.91,2.01$ & \\
\hline CCL2 total & 0.01 & $0.00,2.83$ & 0.111 & 0.01 & $0.00,2.25$ & 0.096 \\
\hline CCL2 stromal & 1.01 & $0.36,2.85$ & 0.984 & 1.37 & $0.52,3.59$ & 0.522 \\
\hline CCL2 epithelial & 0.03 & $0.00,1.59$ & 0.085 & 0.03 & $0.00,1.19$ & 0.062 \\
\hline Fsp1 total & 0.55 & $0.19,1.58$ & 0.266 & 0.60 & $0.22,1.68$ & 0.334 \\
\hline Fsp1 stromal & 0.51 & $0.17,1.49$ & 0.218 & 0.53 & $0.19,1.51$ & 0.235 \\
\hline Fsp1 epithelial & 0.55 & $0.19,1.59$ & 0.273 & 0.78 & $0.29,2.11$ & 0.620 \\
\hline
\end{tabular}

The hazard ratio (HR) was calculated from the Cox proportional hazard regression model. 95\% confidence interval (CI) of the calculated HR is shown. Statistical significance was determined by $P<0.05$.

Ki67 $(<14 \%)$ or mitotic index $(<2)$. Luminal B breast cancers were defined by the expression of ER and/or PR, expression of Her2+ and high Ki67 $(>14 \%)$ or mitotic index $(>2)$. Her2+ breast cancers were identified as ER -, PR - and strongly Her2+, while basal-like breast cancers were defined by the absence of ER, PR and Her2 expression. ${ }^{7-10}$

Luminal A, luminal B and basal-like breast cancers showed significantly higher levels of CCL2 and Fsp1 expression in whole tissues, compared with normal breast tissues (Figure 4a). There were no significant differences in epithelial CCL2 and Fsp1 expression among the different tissue types (Figure 4b). We observed a significant increase in the expression of stromal CCL2 and stromal Fsp1 in luminal A, luminal B and basal-like breast cancers (Figure 4c), compared with normal breast tissues. These data indicate that a significant source of CCL2 and Fsp1 expression originated from the stroma among the different breast cancer subtypes. 



Figure 4 Associations of CCL2 and Fsp1 proteins with molecular subtypes. Expression levels of CCL2 and Fsp1 in whole tissue (a), epithelium (b) and stromal (c) were examined in: normal breast tissues $(n=20)$, luminal A $(n=135)$, luminal B $(n=113)$, Her2+ $(n=42)$, or basal-like $(n=75)$ breast cancers. Statistical analysis was performed using Kruskal-Wallis test with Dunn's post-hoc comparison. Statistical significance was determined by $P<0.05,{ }^{*} P<0.05,{ }^{* *} P<0.001$ and NS $=$ not significant.

CCL2 and Fsp1 were further analyzed for associations with patient outcome among the different breast cancer subtypes. By univariate Cox regression analysis, expression of CCL2 and Fsp1 proteins in whole tissues, stroma or epithelium were not associated with outcome of patients with luminal A, luminal B or Her2 overexpressing breast cancers (Table 3). For patients with basallike breast cancer, total, stromal and epithelial Fsp1 expression did not significantly associate with patient outcome (Table 4, Supplementary Tables S4 and S5). Stromal CCL2 was significantly associated with decreased relapse-free survival (Table 5) by univariate Cox regression analysis.
Furthermore, by multivariate analysis stromal CCL2 (HR =7.51, $P=0.007)$ was associated with a greater hazard than cancer stage $(\mathrm{HR}=2.45$, $P=0.048$ ) (Table 5). Other commonly used prognostic factors, grade and $\mathrm{T}$ stage were not significantly associated with relapse-free survival by univariate analysis. Interestingly, chemotherapy treatment was associated with a higher hazard (HR $=3.73, P=0.034)$ than the absence of chemotherapy, a trend that was also reported in previous studies. ${ }^{40}$ In summary, these data demonstrate that stromal CCL2 expression is associated with decreased recurrence-free survival in patients with basal-like breast cancers. 
Table 3 Associations of CCL2 and Fsp1 expression with outcome for patients with luminal A, luminal B or Her2+ breast cancers

\begin{tabular}{|c|c|c|c|c|c|c|}
\hline \multirow{2}{*}{ Factors } & \multicolumn{3}{|c|}{ Overall survival } & \multicolumn{3}{|c|}{ Recurrence-free survival } \\
\hline & $H R$ & $95 \% C I$ & P-value & $H R$ & $95 \% C I$ & $\mathrm{P}$-value \\
\hline \multicolumn{7}{|l|}{$\operatorname{Lum} A$} \\
\hline CCL2 total & 0.02 & $0.0,32455.8$ & 0.582 & 0.02 & $0.0,5081.37$ & 0.535 \\
\hline CCL2 stromal & 0.33 & $0.01,11.45$ & 0.538 & 1.39 & $0.1,19.65$ & 0.806 \\
\hline CCL2 epithelial & 0.62 & $0.0,2208.55$ & 0.908 & 0.05 & $0.0,250.25$ & 0.493 \\
\hline Fsp1 total & 1.6 & $0.19,13.51$ & 0.665 & 1.13 & $0.15,8.49$ & 0.903 \\
\hline Fsp1 stromal & 0.68 & $0.07,6.21$ & 0.729 & 0.52 & $0.06,4.24$ & 0.543 \\
\hline Fsp1 epithelial & 1.99 & $0.23,16.9$ & 0.529 & 2.41 & $0.35,16.61$ & 0.371 \\
\hline \multicolumn{7}{|l|}{$\operatorname{LumB}$} \\
\hline CCL2 total & 0.02 & $0.0,116.9$ & 0.369 & 0.01 & $0.0,48.28$ & 0.269 \\
\hline CCL2 stromal & 0.59 & $0.1,3.68$ & 0.575 & 0.54 & $0.09,3.25$ & 0.499 \\
\hline CCL2 epithelial & 0.03 & $0.0,11.69$ & 0.253 & 0.03 & $0.0,8.67$ & 0.23 \\
\hline Fsp1 total & 0.76 & $0.11,5.25$ & 0.779 & 0.83 & $0.13,5.47$ & 0.844 \\
\hline Fsp1 stromal & 0.92 & $0.13,6.32$ & 0.932 & 0.73 & $0.11,4.85$ & 0.743 \\
\hline Fsp1 epithelial & 0.4 & $0.07,2.4$ & 0.317 & 0.7 & $0.13,3.86$ & 0.684 \\
\hline \multicolumn{7}{|l|}{ Her2+ } \\
\hline CCL2 total & 0 & $0.0,904.47$ & 0.235 & 0 & $0.0,700.93$ & 0.269 \\
\hline CCL2 stromal & 1.01 & $0.06,18.43$ & 0.993 & 9.17 & $0.24,350.51$ & 0.233 \\
\hline CCL2 epithelial & 0.01 & $0.0,427.02$ & 0.372 & 0.01 & $0.0,214.89$ & 0.352 \\
\hline Fsp1 total & 0.15 & $0.01,1.98$ & 0.151 & 0.3 & $0.03,3.36$ & 0.328 \\
\hline Fsp1 stromal & 0.16 & $0.01,2.39$ & 0.183 & 0.39 & $0.03,5.59$ & 0.485 \\
\hline Fsp1 epithelial & 0.37 & $0.03,5.11$ & 0.459 & 0.42 & $0.03,5.04$ & 0.49 \\
\hline
\end{tabular}

Associations of CCL2 and Fsp1 with overall survival and recurrence-free survival were determined using univariate Cox Regression analysis. 95\% confidence interval (CI) of the calculated hazard ratio (HR) is shown. Statistical significance was determined by $P<0.05$.

Table 4 Associations of CCL2 and Fsp1 expression with patient outcome in basal-like breast cancers

\begin{tabular}{|c|c|c|c|c|c|c|}
\hline \multirow{2}{*}{ Factors } & \multicolumn{3}{|c|}{ Overall survival } & \multicolumn{3}{|c|}{ Recurrence-free survival } \\
\hline & $H R$ & $95 \% C I$ & $\mathrm{P}$-value & $H R$ & $95 \% C I$ & $\mathrm{P}$-value \\
\hline CCL2 total & 0.05 & $0.0,764.46$ & 0.544 & 0.06 & $0.0,1103.32$ & 0.573 \\
\hline CCL2 stromal & 1.61 & $0.55,4.76$ & 0.386 & 5.28 & $1.31,21.25$ & 0.019 \\
\hline CCL2 epithelial & 0.02 & $0.0,69.49$ & 0.333 & 0.01 & $0.0,38.31$ & 0.246 \\
\hline Fsp1 total & 0.13 & $0.02,1.19$ & 0.071 & 0.28 & $0.04,2.31$ & 0.239 \\
\hline Fsp1 stromal & 0.15 & $0.02,1.31$ & 0.086 & 0.31 & $0.04,2.28$ & 0.25 \\
\hline Fsp1 epithelial & 0.36 & $0.03,4.16$ & 0.417 & 0.66 & $0.06,7.27$ & 0.734 \\
\hline
\end{tabular}

Associations of CCL2 and Fsp1 with overall survival and recurrence-free survival were determined using univariate Cox regression analysis. 95\% confidence interval (CI) of the estimated hazard ratio (HR) is shown. Statistical significance was determined by $P<0.05$.

\section{Discussion}

Despite the importance of the stroma in animal models of breast cancer, the use of stromal biomarkers in the clinical setting remains to be validated. Using a novel Image J approach, we detected increased CCL2 and Fsp1 expression in breast tumors. This approach was validated by visual scoring. Furthermore, we report that stromal CCL2 expression correlates with worse relapse-free survival in basal-like breast cancer and was independent of stromal Fsp1 or other commonly recognized prognostic factors, such as stage.

In our studies, we observed several unexpected trends. We found that chemotherapy was associated with decreased overall survival and decreased relapse-free survival. According to univariate analysis in the overall cohort of invasive ductal carcinomas, chemotherapy was associated with decreased overall survival and decrease relapse-free survival (Table 2). This association was lost when adjusted for other variables ( $\mathrm{T}$ stage, $\mathrm{N}$ stage, grade, ER status, PR status; Supplementary Table S6). These results indicate that association between chemotherapy and patient outcome were affected by these other variables. When we analyzed basal-like breast cancers, multivariate analysis revealed that chemotherapy was still significantly associated with decreased overall survival and relapse-free survival (Table 5). There are several possibilities that may account for this trend in basal-like breast cancer. Previous studies showed that chemotherapy treatment was 
Table 5 Univariate and multivariate Cox regression analyses on the association of stromal CCL2 expression with recurrence-free survival of patients with basal-like breast cancers

\begin{tabular}{|c|c|c|c|c|c|c|}
\hline \multirow{2}{*}{ Factors } & \multicolumn{3}{|c|}{ Univariate } & \multicolumn{3}{|c|}{ Multivariate } \\
\hline & $H R$ & $95 \% C I$ & $\mathrm{P}$-value & $H R$ & $95 \% C I$ & P-value \\
\hline Age & 0.99 & $0.96,1.01$ & 0.242 & - & & \\
\hline \multicolumn{7}{|l|}{ Stage } \\
\hline II & 1 & & 0.065 & 1 & & 0.048 \\
\hline III & 1.88 & $0.96,3.67$ & & 2.45 & $1.01,5.95$ & \\
\hline \multicolumn{7}{|l|}{ T stage } \\
\hline $\mathrm{T} 1$ & 1 & & 0.257 & - & & \\
\hline $\mathrm{T} 2$ & 2.54 & $0.75,8.57$ & & - & & \\
\hline T3 & 3.90 & $1.03,14.75$ & & - & & \\
\hline $\mathrm{T} 4$ & 2.56 & $0.57,11.50$ & & - & & \\
\hline \multicolumn{7}{|l|}{ Grade } \\
\hline I & 0 & $0.0,0.0$ & 0.958 & - & & \\
\hline II & 1 & & & - & & \\
\hline III & 0.9 & $0.44,1.84$ & & - & & \\
\hline \multicolumn{7}{|c|}{ Chemotherapy } \\
\hline No & 1 & & 0.015 & 1 & & 0.034 \\
\hline Yes & 2.98 & $1.23,7.19$ & & 3.73 & $1.1,12.58$ & \\
\hline \multicolumn{7}{|c|}{ Radiation therapy } \\
\hline No & 1 & & 0.102 & - & & \\
\hline Yes & 1.8 & $0.89,3.62$ & & - & & \\
\hline \multicolumn{7}{|c|}{ Hormone therapy } \\
\hline No & 1 & & 0.656 & - & & \\
\hline Yes & 0.82 & $0.34,1.98$ & & - & & \\
\hline $\begin{array}{l}\text { CCL2 } \\
\text { stromal }\end{array}$ & 5.28 & $1.31,21.25$ & 0.019 & 7.51 & $1.72,32.91$ & 0.007 \\
\hline
\end{tabular}

Association of CCL2 and recurrence-free survival was determined by Cox univariate or multivariate regression analysis. Hazard ratio (HR) and $95 \%$ confidence interval (CI) of the calculated HR is shown. Statistical significance was determined by $P<0.05$.

associated with a higher hazard than the absence of chemotherapy, a trend that was also reported in a previous study. ${ }^{39}$ There are several possibilities for this trend. We noted that the time of sample collection and diagnosis occurred between 1985 and 1997, prior to the molecular subtyping of breast cancer. Breast cancers diagnosed as invasive ductal carcinomas during this period received a common regimen of chemotherapy and antihormonal therapies. ${ }^{41}$ Development of high grade toxicity, ${ }^{41,42}$ owing in part to combination treatment chemotherapy to tamoxifen, was shown to be associated with poor patient prognosis. ${ }^{41}$ Obesity is another factor affecting chemotherapeutic efficacy and patient outcome; obese patients tend to be undertreated. ${ }^{43,44}$ Recent studies also show that another factor affecting the association between chemotherapy and survival is how early these patients received chemotherapy after surgical intervention. Studies have shown that delays in chemotherapy after surgical intervention negatively correlated with overall survival and relapse-free survival. ${ }^{45-47}$ It is possible that one or more of these factors, which were not included in the pathology reports, may have affected the association between chemotherapy and patient outcome.

We observed that high-level CCL2 expression in the overall cohort was inversely associated with $\mathrm{T}$ stage or $\mathrm{N}$ stage. From these results, it could be interpreted that CCL2 would be associated with good prognosis. We noted that a large number of cases in the overall cohort were luminal $\mathrm{A}$ and $\mathrm{B}$ breast cancers $(n=248)$, which yield a more favorable prognosis than basal-like breast cancer $(n=75) .^{7,8}$ Thus the discrepancy may be due in part to the large proportion of luminal breast cancer cases in our cohort. This proportion was consistent with the overall diagnostic trends in the United States ${ }^{48,49}$ but may have skewed the associations of CCL2 in the overall cohort. Although CCL2 expression was not significant in Her2+ breast cancers in our studies, a recent study of 32 Her2+ breast cancer patients reported that high levels of serum CCL2 associated with favorable outcome in patients vaccinated against Her2 antigen. ${ }^{50}$ High CCL2 expression could predict a more favorable outcome if examined in a therapeutic context. It is also possible that the prognostic value for CCL2 may depend on the breast cancer subtype. In contrast to other breast cancer subtypes, stromal CCL2 expression was associated with poor prognosis in patients with basal-like breast cancer. Stromal CCL2 was associated with a greater hazard than cancer stage or $\mathrm{T}$ stage (Table 5). Yet, stromal CCL2 was inversely associated with overall stage and $\mathrm{T}$ stage in basal-like breast cancer (Supplementary Figure S3). Compared with other breast cancer subtypes, basal-like breast cancers are more likely to recur and be accompanied with poorer prognosis. ${ }^{8,51,52}$ Studies have also shown that commonly used prognostic factors such as tumor size and node status are weakly associated with prognosis in basal-like breast cancers. ${ }^{52}$ Although our studies indicate that overall stage is associated with poor outcome, it is possible that stromal CCL2 expression is a stronger indicator of unfavorable relapse-free survival than overall stage for patients with basal-like breast cancers and may be independent of overall stage or $\mathrm{T}$ stage.

Although CCL2 is expressed in the stroma and epithelium of breast tissues and significantly correlates with Fsp1 expression in the overall cohort, we show that only stromal CCL2 is a significant prognostic factor in recurrent basal-like breast cancers. We did not observe a significant association between CCL2 expression in whole tissues, stroma or epithelium with patient outcome in the overall cohort. These studies contrast with previous studies showing that CCL2 expression correlated with poor patient prognosis regardless of molecular subtype. ${ }^{32,35}$ It is possible that different experimental designs contributed to the different results. We utilized an Image J software approach to 
quantify protein expression in samples collected from patients residing in the United States $(n=427)$. Previous studies utilized smaller sample sizes ( $n=128-150)$, collected from Japanese patients. ${ }^{32,35}$ One study used an ELISA approach to analyze breast tumor samples. ${ }^{35}$ It is possible that regional differences affect the expression of CCL2 in patients. It would be of interest in the future to determine whether demographics affect CCL2 expression in cancer patients. In addition to fibroblastic cells, CCL2 was expressed in infiltrating cells in our patient samples, consistent with previous studies. ${ }^{32,35}$ Previous studies examining CCL2 expression in breast cancer have reported that CCL2 protein is expressed in macrophages and correlate with macrophage recruitment. ${ }^{32,53}$ In another study, CCL2 RNA expression did not correlate with macrophage recruitment in breast cancer. ${ }^{36}$ It is possible that differences in results among published studies were due in part to: sample size, cancer stage, and RNA vs protein expression of CCL2. Our studies indicate the strong possibility that fibroblasts and macrophages together constitute a major source of CCL2 protein expression in breast cancer stroma. Despite the similarities in expression patterns, stromal CCL2 but not stromal Fsp1 was associated with decreased recurrence-free survival of basal-like breast cancer patients. We observed a small but not statistically significant association between total Fsp1 $(\mathrm{HR}=0.13$, $P=0.071)$ and stromal Fsp1 expression $(\mathrm{HR}=0.15$, $P=0.086$ ) with favorable overall survival, opposite results of stromal CCL2. These results further suggest that stromal CCL2 expression is independent of Fsp1 expression in basal-like breast cancers. These results are consistent to one recent study reporting that high expression of Fsp1 in the stroma correlated with favorable overall survival and increased relapse-free survival in the overall cohort in univariate analyses. ${ }^{54}$ It is possible that CCL2 correlates with recruitment of macrophages in triple-negative breast cancer. Multiple macrophage subtypes have been reported in breast cancer, including the presence of M2-like macrophages. ${ }^{55}$ To clearly determine the relevance of CCL2 expression and macrophage recruitment and activity in triple-negative breast cancer, it would be necessary to perform immunostaining for markers identifying these macrophage subtypes, including M1- and M2-like cells, and compare their expression patterns with a panmacrophage marker. ${ }^{55-57}$ These studies are beyond the scope of this manuscript but are of interest in the future.

In summary, our studies demonstrate an association between poor outcome in basal-like breast cancer and CCL2 expression in the stroma. These studies demonstrate that stromal markers may be useful predicting prognosis for specific breast cancer subtypes.

\section{Acknowledgments}

We thank Wendy (Jianghua) He, PhD and Benford Mafuvadze, PhD (University of Kansas Medical Center) for technical support and insightful scientific discussions. This study was funded by American Cancer Association (RSG-13-182-01-CSM).

\section{Disclosure/conflict of interest}

The authors declare no conflict of interest.

\section{References}

1 Torre LA, Bray F, Siegel RL et al. Global cancer statistics 2012. CA Cancer J Clin 2015;65:87-108.

2 Bombonati A, Sgroi DC. The molecular pathology of breast cancer progression. J Pathol 2011;223: 307-317.

3 Harkins B, Geyer CE Jr. Overcoming treatment challenges in advanced breast cancer. Semin Oncol Nurs 2007;23:S10-S16.

4 Amar S, Roy V, Perez EA. Treatment of metastatic breast cancer: looking towards the future. Breast Cancer Res Treat 2009;114:413-422.

5 Koca E, Kuzan TY, Dizdar O et al. Outcomes of locally advanced breast cancer patients with $>/=10$ positive axillary lymph nodes. Med Oncol 2013;30:615.

6 Cheng YC, Ueno NT. Improvement of survival and prospect of cure in patients with metastatic breast cancer. Breast Cancer 2012;19:191-199.

7 Sorlie T, Tibshirani R, Parker J et al. Repeated observation of breast tumor subtypes in independent gene expression data sets. Proc Natl Acad Sci USA 2003;100:8418-8423.

8 Sorlie T, Perou CM, Tibshirani R et al. Gene expression patterns of breast carcinomas distinguish tumor subclasses with clinical implications. Proc Natl Acad Sci USA 2001;98:10869-10874.

9 Cheang MC, Chia SK, Voduc D et al. Ki67 index, HER2 status, and prognosis of patients with luminal B breast cancer. J Natl Cancer Inst 2009;101: 736-750.

10 Aleskandarany MA, Green AR, Benhasouna AA et al. Prognostic value of proliferation assay in the luminal, HER2-positive, and triple-negative biologic classes of breast cancer. Breast Cancer Res 2012;14:R3.

11 Munzone E, Curigliano G, Colleoni M. Tailoring adjuvant treatments for the individual patient with luminal breast cancer. Hematol Oncol Clin North Am 2013;27:703-714, vii-viii.

12 Hudis CA, Gianni L. Triple-negative breast cancer: an unmet medical need. Oncologist 2011;16(Suppl 1): $1-11$.

13 Hasebe T, Mukai K, Tsuda $\mathrm{H}$ et al. New prognostic histological parameter of invasive ductal carcinoma of the breast: clinicopathological significance of fibrotic focus. Pathol Int 2000;50:263-272.

14 Anastassiades OT, Pryce DM. Fibrosis as in indication of time in infiltrating breast cancer and its importance in prognosis. Br J Cancer 1974;29:232-239.

15 Mezi S, Aloise G, Marzullo A et al. Prognostic significance of desmoplasia in breast carcinoma. A preliminary clinical study. G Chir 1997;18:263-268. 
16 Strutz F, Okada H, Lo CW et al. Identification and characterization of a fibroblast marker: FSP1. J Cell Biol 1995;130:393-405.

17 Sugimoto H, Mundel TM, Kieran MW et al. Identification of fibroblast heterogeneity in the tumor microenvironment. Cancer Biol Ther 2006;5:1640-1646.

18 Medrek C, Ponten F, Jirstrom K et al. The presence of tumor associated macrophages in tumor stroma as a prognostic marker for breast cancer patients. BMC Cancer 2012;12:306.

19 Kalluri R, Zeisberg M. Fibroblasts in cancer. Nat Rev Cancer 2006;6:392-401.

$20 \mathrm{Hu} \mathrm{M}$, Yao J, Carroll DK et al. Regulation of in situ to invasive breast carcinoma transition. Cancer Cell 2008;13:394-406.

21 Orimo A, Gupta PB, Sgroi DC et al. Stromal fibroblasts present in invasive human breast carcinomas promote tumor growth and angiogenesis through elevated SDF-1/CXCL12 secretion. Cell 2005;121:335-348.

22 Loeffler M, Kruger JA, Niethammer AG et al. Targeting tumor-associated fibroblasts improves cancer chemotherapy by increasing intratumoral drug uptake. J Clin Invest 2006;116:1955-1962.

23 Brennen WN, Rosen DM, Wang $\mathrm{H}$ et al. Targeting carcinoma-associated fibroblasts within the tumor stroma with a fibroblast activation protein-activated prodrug. J Natl Cancer Inst 2012;104:1320-1334.

24 Abrous-Anane S, Savignoni A, Daveau C et al. Management of inflammatory breast cancer after neoadjuvant chemotherapy. Int J Radiat Oncol Biol Phys 2011;79: 1055-1063.

25 Finak G, Bertos N, Pepin F et al. Stromal gene expression predicts clinical outcome in breast cancer. Nat Med 2008;14:518-527.

26 Allinen M, Beroukhim R, Cai L et al. Molecular characterization of the tumor microenvironment in breast cancer. Cancer Cell 2004;6:17-32.

27 Balkwill FR. The chemokine system and cancer. J Pathol 2012;226:148-157.

28 White GE, Iqbal AJ, Greaves DR. CC chemokine receptors and chronic inflammation-therapeutic opportunities and pharmacological challenges. Pharmacol Rev 2013;65:47-89.

29 Greaves NS, Ashcroft KJ, Baguneid M et al. Current understanding of molecular and cellular mechanisms in fibroplasia and angiogenesis during acute wound healing. J Dermatol Sci 2013;72:206-217.

30 Fang WB, Jokar I, Chytil A et al. Loss of one Tgfbr2 allele in fibroblasts promotes metastasis in MMTV: polyoma middle $\mathrm{T}$ transgenic and transplant mouse models of mammary tumor progression. Clin Exp Metastasis 2011;28:351-366.

31 Fang WB, Jokar I, Zou A et al. CCL2/CCR2 chemokine signaling coordinates survival and motility of breast cancer cells through Smad3 protein- and p42/44 mitogen-activated protein kinase (MAPK)-dependent mechanisms. J Biol Chem 2012;287:36593-36608.

32 Fujimoto H, Sangai T, Ishii G et al. Stromal MCP-1 in mammary tumors induces tumor-associated macrophage infiltration and contributes to tumor progression. Int J Cancer 2009;125:1276-1284.

33 Qian BZ, Li J, Zhang $\mathrm{H}$ et al. CCL2 recruits inflammatory monocytes to facilitate breast-tumour metastasis. Nature 2011;475:222-225.

34 Hembruff SL, Jokar I, Yang L et al. Loss of transforming growth factor-beta signaling in mammary fibroblasts enhances CCL2 secretion to promote mammary tumor progression through macrophage-dependent and -independent mechanisms. Neoplasia 2010;12:425-433.

35 Ueno T, Toi M, Saji H et al. Significance of macrophage chemoattractant protein-1 in macrophage recruitment, angiogenesis, and survival in human breast cancer. Clin Cancer Res 2000;6:3282-3289.

36 Valkovic T, Fuckar D, Stifter S et al. Macrophage level is not affected by monocyte chemotactic protein-1 in invasive ductal breast carcinoma. J Cancer Res Clin Oncol 2005;131:453-458.

37 Fang WB, Yao M, Jokar I et al. The CCL2 chemokine is a negative regulator of autophagy and necrosis in luminal B breast cancer cells. Breast Cancer Res Treat 2015;150: 309-320.

38 Zou A, Lambert D, Yeh $\mathrm{H}$ et al. Elevated CXCL1 expression in breast cancer stroma predicts poor prognosis and is inversely associated with expression of TGF-beta signaling proteins. BMC Cancer 2014;14: 781.

39 Rizzardi AE, Johnson AT, Vogel RI et al. Quantitative comparison of immunohistochemical staining measured by digital image analysis versus pathologist visual scoring. Diagn Pathol 2012;7:42.

40 Abadi A, Yavari P, Dehghani-Arani M et al. Cox models survival analysis based on breast cancer treatments. Iran J Cancer Prev 2014;7:124-129.

41 Morrow M, Krontiras $H$. Who should not receive chemotherapy? Data from American databases and trials. J Natl Cancer Inst Monogr 2001;109-113.

42 Maskarinec G, Pagano I, Lurie G et al. Factors affecting survival among women with breast cancer in Hawaii. J Womens Health (Larchmt) 2011;20:231-237.

43 Griggs JJ, Sorbero ME, Lyman GH. Undertreatment of obese women receiving breast cancer chemotherapy. Arch Intern Med 2005;165:1267-1273.

44 Hunter RJ, Navo MA, Thaker PH et al. Dosing chemotherapy in obese patients: actual versus assigned body surface area (BSA). Cancer Treat Rev 2009;35: 69-78.

45 Chavez-MacGregor M, Clarke CA, Lichtensztajn DY et al. Delayed initiation of adjuvant chemotherapy among patients with breast cancer. JAMA Oncol 2015;2:322-329.

46 Bleicher RJ, Ruth K, Sigurdson ER et al. Time to surgery and breast cancer survival in the United States. JAMA Oncol 2015;2:330-339.

47 Waks AG, King TA, Winer EP. Timeliness in breast cancer treatment-the sooner, the better. JAMA Oncol 2015;2:302-304.

48 Kwan ML, Kushi LH, Weltzien E et al. Epidemiology of breast cancer subtypes in two prospective cohort studies of breast cancer survivors. Breast Cancer Res 2009;11:R31.

49 Rezai M, Kellersmann S, Knispel S et al. Translating the concept of intrinsic subtypes into an oncoplastic cohort of more than 1000 patients - predictors of recurrence and survival. Breast 2015;24:384-390.

50 Dehqanzada ZA, Storrer CE, Hueman MT et al. Correlations between serum monocyte chemotactic protein-1 levels, clinical prognostic factors, and HER-2/neu vaccine-related immunity in breast cancer patients. Clin Cancer Res 2006;12:478-486.

51 Kim MJ, Ro JY, Ahn SH et al. Clinicopathologic significance of the basal-like subtype of breast cancer: a comparison with hormone receptor and Her2/neuoverexpressing phenotypes. Hum Pathol 2006;37: 1217-1226. 
52 Dent R, Trudeau M, Pritchard KI et al. Triple-negative breast cancer: clinical features and patterns of recurrence. Clin Cancer Res 2007;13:4429-4434.

53 Saji H, Koike M, Yamori T et al. Significant correlation of monocyte chemoattractant protein-1 expression with neovascularization and progression of breast carcinoma. Cancer 2001;92:1085-1091.

54 Park SY, Kim HM, Koo JS. Differential expression of cancer-associated fibroblast-related proteins according to molecular subtype and stromal histology in breast cancer. Breast Cancer Res Treat 2015;149:727-741.
55 Laoui D, Movahedi K, Van Overmeire E et al. Tumorassociated macrophages in breast cancer: distinct subsets, distinct functions. Int J Dev Biol 2011;55: 861-867.

56 Stoger JL, Gijbels MJ, van der Velden S et al. Distribution of macrophage polarization markers in human atherosclerosis. Atherosclerosis 2012;225: 461-468.

57 Sousa S, Brion R, Lintunen $\mathrm{M}$ et al. Human breast cancer cells educate macrophages toward the M2 activation status. Breast Cancer Res 2015;17:101.

Supplementary Information accompanies the paper on Modern Pathology website (http://www.nature.com/ modpathol) 